\title{
CONCAVITY OF MAGNETIZATION FOR A CLASS OF EVEN FERROMAGNETS ${ }^{1}$
}

\author{
BY RICHARD S. ELLIS
}

Communicated by Daniel Stroock, May 5, 1975

1. Introduction. Let $E$ be the set of even probability measures which satisfy $\int \exp \left(k x^{2}\right) \rho(d x)<\infty$ for all $k \geqslant 0$ sufficiently small. Given an integer $N \geqslant 1$, real numbers $h \geqslant 0$ and $J_{i j} \geqslant 0,1 \leqslant i \leqslant j \leqslant N$, and measures $\rho_{i} \in E$, $1 \leqslant i \leqslant N$, we define $\left[11\right.$, p. 273] real-valued random variables $X_{i}, 1 \leqslant i \leqslant N$, with the joint distribution

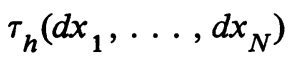

$$
=\frac{\exp \left(\Sigma_{1 \leqslant i<j \leqslant N} J_{i j} x_{i} x_{j}+h \Sigma_{1 \leqslant i \leqslant N} x_{i}\right) \rho_{1}\left(d x_{1}\right) \ldots \rho_{N}\left(d x_{N}\right)}{Z(h)} .
$$

$Z(h)$, the partition function, is given by the formula

$$
Z(h)=\int \underset{R^{N}}{\ldots} \int \exp \left(\sum J_{i j} x_{i} x_{j}+h \sum_{x_{i}}\right) \rho_{1}\left(d x_{1}\right) \cdots \rho_{N}\left(d x_{N}\right) .
$$

The $J_{i j}$ are assumed to be so small that the integral in (2) converges for all $h \geqslant 0$. The inequalities we discuss are to hold for all $h \geqslant 0$ and all $J_{i j} \geqslant 0$ subject only to this restriction. The choice of $\rho_{i}$ as the Bernoulli measure $b(d x)=$ $1 / 2(\delta(x-1)+\delta(x+1))$ gives the classical Ising model.

We define the average magnetization per site, $m(h)$, by the formula

$$
m(h)=\frac{1}{N} \frac{d}{d h} \ln Z(h)=\frac{1}{N} \sum_{i=1}^{N} E\left\{X_{i}\right\}
$$

and consider inequalities on $m(h)$ and its derivatives. While the inequalities $m(h) \geqslant 0, d m(h) / d h \geqslant 0$ hold for any $\rho_{i} \in E[7$, pp. 76-77], the concavity of $m(h)$, i.e.

$$
d^{2} m(h) / d h^{2} \leqslant 0
$$

requires that further restrictions be placed on the $\rho_{i}$. Essentially, (4) is known to hold only in the Ising case and in models which can be built out of Ising models in a suitable way [4], [6]. Measures for which (4) fails are known [6]. 26A51.

AMS (MOS) subject classifications (1970). Primary 82A05, 60K35; Secondary 60E05,

Key words and phrases. Even ferromagnet, partition function, average magnetization per site, GHS inequality.

${ }^{1}$ Research supported in part by National Science Foundation Grant GP-28576. 
The usual approach to (4) is first to prove the stronger (GHS) inequalities [5]

$$
\frac{\partial^{3}}{\partial h_{i} \partial h_{j} \partial h_{k}} \ln Z\left(h_{1}, \ldots, h_{N}\right) \leqslant 0, \quad \text { all } 1 \leqslant i, j, k \leqslant N, h_{i} \geqslant 0
$$

where

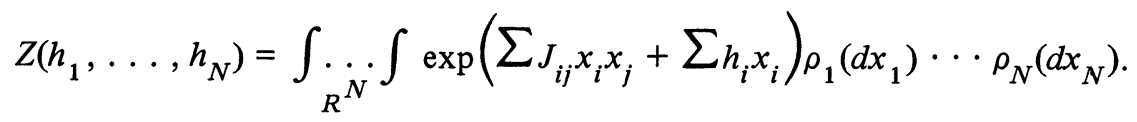

Instead, we shall prove (4) directly for many new measures using a technique which reduces consideration to the case $N=1$. Afterwards, we shall return to (5).

We state two implications of these inequalities. The first shows that the requirement that the $\rho_{i}$ in (1) have Gaussian falloff is only an apparent restriction.

THEOREM 1. Let $\rho$ be an even probability measure satisfying $\int \exp (k x) \rho(d x)$ $<\infty$ for all $k \geqslant 0$. Assume that (4) holds for $N=1$ (set $\rho_{1}=\rho$ ). Then $\rho$ is in $E$.

The next theorem (known for fourth degree polynomial $V$ [3], [10]) on the spectrum of certain differential operators is a striking consequence of (5).

THEOREM 2. Let $V(x)$ be an entire function with the expansion

(6) $V(x)=\sum_{k=1}^{\infty} a_{k} x^{2 k}, \quad a_{k} \geqslant 0$ for $k \geqslant 2, \quad a_{1}$ real $\left(a_{1}>0\right.$ if all $\left.a_{k}=0\right)$.

Let $E_{1}, E_{2}, E_{3}$, be the three smallest eigenvalues of the differential operator $-1 / 2 d^{2} / d x^{2}+V(x)$ on $L^{2}\left(R^{1} ; d x\right)$. Then $E_{3}-E_{2} \geqslant E_{2}-E_{1}$.

By Theorems 4 and 5 below, we shall see that (5) is satisfied for the measures

$$
\rho_{i}(d x)=c \exp (-V(x)) d x, \quad c \text { a normalization constant, }
$$

if $V$ is as in (6). This is the main ingredient needed to prove Theorem 2 [10].

2. The class $G_{-}$. Below, we define a subset $G_{-}$of measures in $E$ for which we have the following result.

THEOREM 3. If $\rho_{1}, \ldots, \rho_{N} \in G_{-}$, then (4) holds.

For the proof, we use a closure property of $G_{-}$in order to reduce to the case $N=1$. We call this property the closure of $G_{-}$under ferromagnetic unions.

(C) Let $Y_{1}, \ldots, Y_{N}$ be real-valued random variables with joint distribution $\tau_{0}($ see $(1))$. Let $F_{0}$ be the class of all distributions of sums $\Sigma_{1 \leqslant i \leqslant N} r_{i} Y_{i}$ 
for arbitrary choice of $N \geqslant 1, r_{i} \geqslant 0, J_{i j} \geqslant 0$, and $\rho_{1}, \ldots, \rho_{N} \in G_{-}$. Then $F_{0} \subseteq G$.

The partition function $Z(H)$ in (2) can be written as

$$
Z(h)=Z(0) E\left\{\exp \left(h \sum_{1 \leqslant i \leqslant N} Y_{i}\right)\right\}
$$

i.e., $m(h)$ is related to the average magnetization $\widetilde{m}(h)$ for a single site system (with spin $\Sigma_{1 \leqslant i \leqslant N} Y_{i}$ at the single site) by the formula $m(h)=N^{-1} \tilde{m}(h)$. Hence, Theorem 3 for general $N$ is a consequence of (C) once we have proved Theorem 3 for $N=1$. We do the latter in $\S 3$.

The next theorem indicates which measures belong to $G_{-}$.

THEOREM 4. G_ contains the Bernoulli measure $b(d x)$ and all measures of

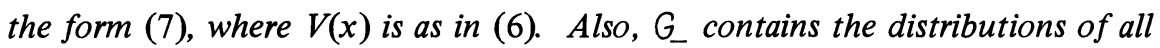
weak limits of $Y^{(N)} \in F_{0}$ which satisfy $\sup _{N} E\left\{\left(Y^{(N)}\right)^{2}\right\}<\infty$.

The first part of Theorem 4 will be proved after $G_{-}$is defined.

Definition. Given $\rho \in E$, let $W_{1}, \ldots, W_{4}$ be four independent copies of a random variable distributed by $\rho$. The vector $\vec{m}=\left(m_{1}, \ldots, m_{4}\right)$, where each $m_{i}$ is a nonnegative integer, is said to be odd if each $m_{i}$ is odd. Let $W=$ $\left(W_{1}, \ldots, W_{4}\right)$; take $A$ to be the orthogonal matrix

and define

$$
2^{-1}\left(\begin{array}{rrrr}
1 & 1 & 1 & 1 \\
-1 & 1 & -1 & 1 \\
-1 & -1 & 1 & 1 \\
1 & -1 & -1 & 1
\end{array}\right)
$$

$$
(A \vec{W})_{i}=\sum_{j=1}^{4} A_{i j} W_{j}, \quad(A \vec{W})^{\vec{m}}=(A \vec{W})_{1}^{m} 1 \cdots(A \vec{W})_{4}^{m}{ }^{4}, \quad \mu_{\rho}(\vec{m})=E\left\{(A \vec{W})^{\vec{m}}\right\} .
$$

We define

$$
G_{-}=\left\{\rho: \rho \in E \text { and } \mu_{\rho}(\vec{m}) \leqslant 0 \text { for all } \vec{m} \text { odd }\right\} .
$$

The condition that $\mu_{\rho}(\vec{m}) \leqslant 0$ for all $\vec{m}$ odd implies an infinite string of inequalities satisfied by the moments of a measure $\rho \in G_{-}$. We refer the reader to [8] and [9], where other moment inequalities are derived for measures which satisfy the Lee-Yand theorem.

To show that $b(d x) \in G_{-}$, consider

$$
\begin{aligned}
\mu_{b}(\vec{m})=\frac{1}{16} \sum_{x_{i}= \pm 1}\left(x_{1}+x_{2}+x_{3}+x_{4}\right)^{m}\left(-x_{1}+x_{2}-x_{3}+x_{4}\right)^{m} 2 \\
\cdot\left(-x_{1}-x_{2}+x_{3}+x_{4}\right)^{m}\left(x_{1}-x_{2}-x_{3}+x_{4}\right)^{m} .
\end{aligned}
$$

Each of the 16 summands is either negative or zero according to whether an odd number or an even number of the $x_{i}$ equal +1 .

Given a measure $\rho$ as in (7), the joint distribution of the random vector 
$A \vec{W}$ has the form

$$
\exp \left(-f\left(z_{1}, \ldots, z_{4}\right)\right) \exp \left(g\left(z_{1}, \ldots, z_{4}\right)\right) d z_{1} \cdots d z_{4},
$$

where $f$ is an odd function of each $z_{i}$ and $f \geqslant 0$ when each $z_{i} \geqslant 0$ and $g$ is an even function of each $z_{i}$. Greater weight is thus given to those values of $z_{1}, \ldots$ $z_{4}$ where an odd number of the $z_{i}$ are negative than where an even number of the $z_{i}$ are negative. From this, it can be shown that $\rho \in G_{-}$.

We also have a characterization of Gaussian measures in terms of $G_{\text {_ }}$.

THEOREM 5. Given $\rho \in E$, the numbers $\mu_{\rho}(\vec{m})=0$ for all $\vec{m}$ odd if and only if $\rho$ is an even Gaussian measure.

Inequality (5) holds under the same hypothesis as (4).

THEOREM 6. If $\rho_{1}, \ldots, \rho_{N} \in G_{-}$, then (5) holds.

The proof makes use of multivariate versions of the $G_{-}$inequalities. Let $Y_{i}^{(j)}, 1 \leqslant j \leqslant 4$, be independent copies of $Y_{i}$ (see (C)) and define $\vec{Y}_{i}=\left(Y_{i}^{(1)}\right.$, $\left.\ldots, Y_{i}^{(4)}\right)$. Then

$$
E\left\{\left(A Y_{1}\right)^{\vec{m}} \ldots\left(A \vec{Y}_{N}\right)^{\vec{m}} N_{1} \leqslant 0\right.
$$

whenever $\rho_{1}, \ldots, \rho_{N} \in G_{-}$and $\vec{m}_{1}+\ldots+\vec{m}_{N}$ is odd.

3. Proof of Theorem 3 for $N=1$. Given $\rho \in G_{\text {, }}$, we write $Z(h)=$ $\int \exp (h x) \rho(d x), h \geqslant 0$. We have (' denotes $\left.d / d h\right)$

$$
\begin{aligned}
(\ln Z)^{\prime \prime \prime}= & \left(Z^{2} Z^{\prime \prime \prime}-3 Z Z^{\prime} Z^{\prime \prime}+2\left(Z^{\prime}\right)^{3}\right) / Z^{3} \\
= & \frac{1}{Z^{4}} \int_{R^{4}} \ldots \int\left[\frac{\partial^{3}}{\partial h_{1}^{3}}-3 \frac{\partial^{3}}{\partial h_{1}^{2} \partial h_{2}}+2 \frac{\partial^{3}}{\partial h_{1} \partial h_{2} \partial h_{3}}\right] \\
& \left.\cdot e^{\langle\vec{h}, \vec{x}\rangle} \rho\left(d x_{1}\right) \ldots \rho\left(d x_{4}\right)\right|_{h_{i}=h},
\end{aligned}
$$

where $\vec{h}=\left(h_{1}, \ldots, h_{4}\right), \vec{x}=\left(x_{1}, \ldots, x_{4}\right)$, and $\langle\cdot, \cdot\rangle$ is the $R^{4}$ inner product. Define $\vec{s}=\left(s_{1}, \ldots, s_{4}\right)=\vec{h} A^{t}$. An easy calculation [1, Appendix] shows that the last integral equals

$$
\left.\frac{2}{Z^{4}} \int \ldots \int_{R^{4}} \frac{\partial^{3}}{\partial s_{2} \partial s_{3} \partial s_{4}} e^{\langle\vec{s}, A \vec{x}\rangle} \rho\left(d x_{1}\right) \cdots \rho\left(d x_{4}\right)\right|_{h_{i}=h} .
$$

Expanding the exponential and carrying out the integration, we find

$$
\begin{aligned}
& (\ln Z)^{\prime \prime \prime}=\frac{2}{Z^{4}} \sum_{n=0}^{\infty} \sum_{m_{1}+\ldots+m_{4}=n} \frac{m_{2} m_{3} m_{4}}{m_{1} ! \cdots m_{4} !} \\
& \left.\cdot \mu_{\rho}(\vec{m}) s_{1}^{m}{ }_{1} s_{2}^{m_{2}-1} s_{3}^{m_{3}-1} s_{4}^{m_{4}}{ }^{-1}\right|_{h_{i}=h} .
\end{aligned}
$$


But when each $h_{i}=h$, then $s_{1}=2 h, s_{2}=s_{3}=s_{4}=0$. Also, $\mu_{\rho}((k, 1,1,1))$ can be shown to be zero unless $k$ is odd. Hence

$$
(\ln Z)^{\prime \prime \prime}=\frac{2}{Z^{4}} \sum_{k \text { odd } ; k \geqslant 0} \frac{(2 h)^{k}}{k !} \mu_{\rho}((k, 1,1,1)),
$$

which is negative since $\rho \in G_{-}$and $h \geqslant 0$. This completes the proof.

In this proof, we did not need the full force of the assumption that $\rho \in G_{-}$; viz., that $\mu_{\rho}(\vec{m}) \leqslant 0$ for all $\vec{m}$ odd. However, the latter is needed to prove Theorem 6. Also, the set of measures $\vec{\rho}$ for which $\mu_{\rho}((k, 1,1,1)) \leqslant 0$ for all $k$ odd is not necessarily closed under ferromagnetic unions.

REMARK. Proofs and related matter will appear in [2] .

\section{REFERENCES}

1. R. S. Ellis and J. L. Monroe, A simple proof of the GHS and further inequalities, Comm. Math. Phys. 41 (1975), 33-38.

2. The GHS and other correlation inequalities for even ferromagnets (in preparation).

3. J. Glimm, A. Jaffe, and T. Spencer, The particle structure of the weakly coupled $P(\varphi)_{2}$ model and other applications of high temperature expansions. Part I: Physics of quantum field models, Constructive Quantum Field Theory, G. Velo and A. S. Wightman (editors), Springer-Verlag, New York, 1973, pp. 133-198.

4. R. B. Griffiths, Rigorous results for Ising ferromagnets of arbitrary spin, J. Mathematical Phys. 10 (1969), $1559-1565$. MR 41 \#1338.

5. R. B. Griffiths, C. A. Hurst and S. Sherman, Concavity of magnetization of an Ising ferromagnet in a positive external field, J. Mathematical Phys. 11 (1970), 790-795. MR 42 \#1412.

6. R. B. Griffiths and B. Simon, The $\left(\varphi^{4}\right)_{2}$ field theory as a classical Ising model, Comm. Math. Phys. 33 (1975), 145-164.

7. A. Ya. Hinčin, Mathematical foundations of statistical mechanics, OGIZ, Moscow, 1943; English transl., Dover, New York, 1949. MR 8, 187; 10, 666.

8. C. M. Newman, Inequalities for Ising models and field theories which obey the Lee-Yang theorem, Comm. Math. Phys. 41 (1975), 1-9.

9. - Moment inequalities for ferromagnetic Gibbs distributions, J. Mathematical Phys. (to appear).

10. B. Simon, Approximation of Feynman integrals and Markov fields by spin systems, Proc. Intl. Congress Math., Vancouver, 1974.

11. - The $P(\varphi)_{2}$ Euclidean (quantum) field theory, Princeton Univ. Press, Princeton, N. J., 1974.

\section{DEPARTMENT OF MATHEMATICS, NORTHWESTERN UNIVERSITY, EVANSTON, ILLINOIS 60201}

Current address: Department of Mathematics and Statistics, University of Massachusetts, Amherst, Massachusetts 01002 